\title{
La francophonie, le français, son génie et son déclin
}

\author{
Luc Pinhas
}

\section{(2) OpenEdition}

\section{Journals}

\section{Édition électronique}

URL : https://journals.openedition.org/dhfles/101

DOI : $10.4000 /$ dhfles.101

ISSN : 2221-4038

\section{Éditeur}

Société Internationale pour l'Histoire du Français Langue Étrangère ou Seconde

\section{Édition imprimée}

Date de publication : 1 janvier 2008

Pagination : 51-61

ISSN : 0992-7654

\section{Référence électronique}

Luc Pinhas, «La francophonie, le français, son génie et son déclin », Documents pour I'histoire du français langue étrangère ou seconde [En ligne], 40/41 | 2008, mis en ligne le 18 décembre 2010, consulté le 27 mai 2021. URL : http://journals.openedition.org/dhfles/101 ; DOI : https://doi.org/ 10.4000/dhfles. 101

Ce document a été généré automatiquement le 27 mai 2021

(C) SIHFLES 


\title{
La francophonie, le français, son génie et son déclin
}

\author{
Luc Pinhas
}

1 Pour peu qu'on la situe dans un contexte géopolitique large, la genèse du discours sur la francophonie, des années d'après-guerre aux années 1960, semble indéniablement marquée, à tout le moins du point de vue hexagonal, par le sentiment d'un déclin déchirant à admettre pour la communauté concernée - du rayonnement de la langue française dans le monde et, plus généralement de la culture française. Cette appréhension nouvelle d'un "déclassement ${ }^{1}{ }^{~}$ " en cours touche à l'identité française même, tant en France, la langue, la culture, l'État et la nation font partie d'« une expérience unique ", ainsi que le suggérait en 1930 le philologue allemand Ernst-Robert Curtius, dans son Essai sur la France. La tourmente de la décolonisation, au même moment, ne peut bien évidemment qu'aviver les craintes d'un recul.

2 Corrélativement, la montée en puissance, non seulement politique, militaire et économique, mais aussi désormais culturelle, des États-Unis d'Amérique, ne cesse plus à cette époque d'inquiéter les élites françaises, tout autant qu'elle les fascine, de sorte qu'au désarroi de la perte de l'Empire français s'ajoutent alors les affres d'une colonisation rampante de la part d'un pays avec lequel, il est vrai, les relations sont de longue date ambivalentes et ne cesseront de le rester. De fait, les deux nations ont la prétention implicite de proposer au monde deux modèles rivaux d'universel qui les placent en position de concurrence redoutable.

3 C'est dans ce contexte, nous semble-t-il, qu'il convient d'apprécier la résurgence, dans les écrits de Senghor et dans le numéro célèbre de novembre 1962 de la revue Esprit, significativement intitulé "Le français, langue vivante», des termes de "francophone " et de "francophonie", forgés, ou à tout le moins abondamment utilisés, au tournant du XIX ${ }^{e}$ au XX ${ }^{e}$ siècle, par Onésime Reclus. Or, l'on feint parfois d'oublier que le travail du géographe se produit à un autre moment où la France s'interroge sur sa grandeur et s'inscrit dans une réflexion sur la question coloniale, comme l'on ignore trop souvent l'obsession démographique qui présidait à une pensée hantée par le spectre de la décadence². 
4 Le recours conjugué, dans le discours fondateur de la francophonie contemporaine, à une double mythologie de la langue, d'une part à la mythologie «rivarolienne » du génie de la langue française, d'autre part à la mythologie révolutionnaire du français, langue des droits de l'homme et de l'universel, peut ainsi apparaître, quitte à ce qu'il soit actualisé par l'invocation de la "diversité », comme une tentative de redonner son éclat, sinon à la France, du moins à une pensée française qui entend alors manifester clairement sa voix(e), face à celle, grossissante et amplifiée, de l'Amérique, porteuse d'une autre conception de l'universalité.

5 Il s'agit là, du moins, de l'hypothèse que nous souhaitons ici exposer.

\section{La double mythologie du français}

6 L'on s'étonne souvent, à l'étranger surtout, mais parfois aussi en France, de l'appellation même de la Francophonie, c'est-à-dire de l'idée d'un rassemblement de pays, territoires et peuples autour de l'affirmation d'une langue, la langue française en l'occurrence. C'est ignorer à quel point celle-ci, pour ses locuteurs français du moins, est plus qu'une langue. À travers elle, prennent chair une littérature certes, mais aussi une culture, une vision de l'universel et, osons le dire, un destin. Ce lien inextricable peut remonter, si l'on suit les analyses de Renée Balibar, jusqu'au moment originel de la naissance de la " Nation France », c'est-à-dire jusqu'aux serments de Strasbourg de 842 prononcés en langues vulgaires, roman et tudesque, par Charles le Chauve et Louis le Germanique, ou, plus précisément à leur rapport écrit par le moine Nithard dans son Histoire des fils de Louis le Pieux, lequel rapport a d'ailleurs fait dire à Bernard Cerquiglini que «le français national, notre français, ne provient pas d'un terroir, mais de la littérature $»^{3}$. Il se consolide ensuite du thème de la translatio studii (et imperii) qu'utilise, depuis Charles V et Nicolas Oresme au moins, le pouvoir royal pour se renforcer, et selon lequel il y aurait depuis Charlemagne un passage de témoin de la Rome antique à la France, de sorte que le français serait appelé à devenir «le latin des temps modernes ». Son déploiement, encouragé et amplifié, contribuera longtemps, et sans doute jusqu'à aujourd'hui, à entretenir ce que Marc Fumaroli nomme une «mystique » de la langue française. Ce dernier voit au demeurant dans l'institutionnalisation du français, « langue du Roy », et dans l'article 110 de l'ordonnance de Villers-Cotterêts, la naissance du mythe de la clarté grâce auquel «la langue du roi et de la cour va prétendre au rang de 'latin des modernes'«. Il s'agit là précisément de la tâche que Richelieu assigne explicitement à l'Académie française ${ }^{4}$ pour que la translatio studii soit parachevée et consacre la suprématie de la civilisation française, que sa littérature se trouve chargée de manifester au monde. Le mythe du génie de la langue française peut alors prendre son essor pour se nourrir de la philosophie des Lumières et de leur recherche d'un humanisme supérieur qui reposerait sur l'usage d'une raison transparente et transcenderait tous les particularismes culturels. Il trouve juste avant la Révolution française son théoricien par excellence avec Antoine de Rivarol et sa bible avec le Discours sur l'universalité de la langue française qui, par un audacieux coup de plume, détourne le sens de la question posée par l'Académie de Berlin pour passer d'une universalité relative à une universalité absolue.

7 La Révolution française n'abandonne pas la langue, mais la confisque à son tour à son profit. Loin de provoquer une rupture du lien entre la langue et le politique, elle le retourne à ses propres fins. «Langue : se prend aussi quelquefois pour Nation », indique 
la cinquième édition, de 1798, du dictionnaire de l'Académie ${ }^{5}$. Le français devient alors autant le garant de l'unité nationale que la langue universelle de la liberté et des droits de l'homme qui combat le fanatisme et la superstition, une seconde mythologie venant ainsi se superposer à la première. Le rôle premier dévolu à l'école sera désormais de le répandre dans toutes les couches de la population de façon à renforcer l'identité collective française et à assurer l'égalité républicaine, quitte à brider le particularisme des langues régionales au nom de ce que Pierre Bourdieu nomme « un impérialisme de l'universel $»^{6}$. C'est pourquoi la pénétration de l'anglais va-t-elle être ressentie si fortement comme un envahissement et une menace d'acculturation et finira par imposer en 1992 l'inscription constitutionnelle de ce qui jusqu'alors passait pour une évidence, le fait que « la langue de la République est le français ».

8 Il est peu de dire que cette double mythologie, dont l'objectif est bien de mettre en avant la vocation universelle du français, structure le discours de ceux que Christophe Traisnel ${ }^{7}$ nomme "les francophonistes", de la première mais aussi de la deuxième heure. Je ne ferai pas ici une analyse du discours senghorien, ni de celui qui parcourt le numéro d'Esprit de 1962, mais l'un et l'autre sont indéniablement parcourus de cet argumentaire duel. Celui-ci n'en est pas moins présent, au moins sous forme de non-dit, lorsque sont mis en concours, en 1987, de nouveaux "discours sur l'universalité de la francophonie » ou lorsque Dominique Combes étudie les " poétiques francophones $»^{8}$. Il habite tout autant le Nouveau discours sur l'universalité de la langue française de Thierry de Beaucé, paru en 1988. Pour ce dernier, en effet, le français, "en défendant ses mots ", entend participer d'une grande espérance et même, comme autrefois, l'initier et la guider comme si, à nouveau, «il revenait à la France de prendre la tête d'un mouvement qui rassemble en les constatant toutes les civilisations ${ }^{9}$. Le modèle universel ainsi proposé doit toutefois être actualisé pour prendre en compte la nouvelle donne internationale et l'avancée de la globalisation économique. Le français, que la France partage désormais avec tous les pays francophones, auxquels elle en a fait don et qui contribuent à le rendre multiple, se voit alors mis au service d'un projet démocratique qui ne sacrifierait pas le culturel sur l'autel du libéralisme économique et tenterait, face aux velléités uniformisantes de l'hégémonisme américain, de préserver la diversité des identités tout en déjouant le piège du communautarisme. C'est pourquoi le nouveau discours de la Francophonie entend proposer aujourd'hui une alternative à l'idéologie techniciste d'une communication envisagée dans sa seule dimension fonctionnelle pour en développer les potentialités normatives ${ }^{10}$ et opérer ainsi une réconciliation de la raison et de la culture, qui ressemble fort au demeurant à la «symbiose » qu'appelait de ses vœux Senghor.

\section{Le discours originel : Onésime Reclus}

9 Si l'on se penche à présent sur le discours originel de la francophonie tel qu'il apparaît dans les écrits d'Onésime Reclus, une autre ambivalence se remarque. L'œuvre du frère méconnu d'Élisée Reclus est en effet très largement consacrée à une réflexion sur le développement du second Empire colonial français et sur les moyens pour la France de recouvrer une grandeur menacée après la défaite de Sedan et la perte de l'AlsaceLorraine. Il se fait donc, au cours des années 1880, l'un des théoriciens de la politique coloniale alors défendue par Jules Ferry. Les premières occurrences connues des termes «francophone» et «francophonie » apparaissent précisément dès 1880, dans un 
ouvrage intitulé France, Algérie et colonies, dans lequel le géographe, après avoir rappelé l'essor de la langue française au cours du passé, s'attache à établir le décompte minutieux des locuteurs de la langue française à travers le monde, autrement dit des «francophones ». Le total auquel il aboutit, cependant, ne le satisfait guère, lui qui ne cessera plus de confronter, inlassablement jusqu'à sa mort en 1916, le nombre de «Français de langue » à celui des locuteurs des autres principales langues européennes, c'est-à-dire à ses yeux civilisées et civilisatrices. Cette obsession est particulièrement visible dans Lâchons l'Asie, prenons l'Afrique (1904), dont le sous-titre significatif pose les questions : «Où renaître ? Et comment durer? ».

10 La première question renvoie d'abord, bien évidemment, aux « malheurs » de la France après la défaite face à la Prusse et à la déliquescence de « cette vieille race qui sentait le cadavre au printemps de $1871 »^{11}$, bien qu'elle ait su depuis, pense Reclus, se ressaisir et se donner de nouveaux espoirs. Mais elle fait tout autant référence à l'Empire perdu, aux anciennes possessions sur le continent américain que les gouvernants précédents ont négligées et qui se trouvent désormais soumises à l'emprise de la langue anglaise. La réponse à apporter, quant à elle, se trouve contenue dans le titre et, puisque «la France amoindrie par son peu de natalité ne peut embrasser le monde [...] il lui faut renoncer à ce qui n'est pas l'Afrique $»^{12}$. Il ne s'agit pas, pourtant, uniquement de renaître, il faut également s'assurer d'une éternelle durée et celle-ci ne peut être le fait que d'une assimilation bien menée. Or celle-ci, on l'aura compris, passe par la langue. Pour Onésime Reclus, qui ne croit fondamentalement pas aux races, à l'inverse d'un Jules Ferry, la langue est bel et bien le fer de lance de l'assimilation recherchée, le ciment d'une communauté de pensée, le creuset qui «amalgame à la longue, en une vaste nation francisante, les peuples inféodés ${ }^{13}$, règle les pensées et les activités, fusionne les éléments divers en une civilisation commune. Pour le signifier autrement, l'idée-force qui parcourt toute l'œuvre de Reclus et lui assure sa cohérence, celle dans laquelle se trouve sans aucun doute le substrat de l'entreprise francophone, réside dans une conviction inébranlable, et tellement française, que « la langue fait le peuple »:

Dès qu'une langue a "coagulé » un peuple, tous les éléments «raciaux» de ce peuple se subordonnent à cette langue. C'est dans ce sens qu'on a dit : la langue fait le peuple (lingua gentem facit) ${ }^{14}$.

11 La quête des voies de l'assimilation par la langue entraîne alors, comme tout naturellement, à se tourner vers un grand modèle - auquel se réfèrent également, certes, nombre d'autres auteurs de la période : le modèle de l'Empire romain. L'objectif est en effet bel et bien de "faire en Afrique ce que Rome fit dans le monde ancien ${ }^{15} \mathrm{et}$, pour ce faire, de suivre les voies alors adoptées, que Reclus détaille longuement ${ }^{16}$. Ainsi ce dernier n'a-t-il de cesse que de tracer les perspectives d'un renouveau français qui coïncide avec la mission civilisatrice de la France, nouvelle Rome des temps modernes, et qui renvoie à l'un des thèmes majeurs de la mythologie française.

\section{Une relation ambiguë à « l'Amérique »}

L'après-Seconde-Guerre-mondiale voit derechef la France et la culture française s'interroger sur leur grandeur. Les bouleversements que connaît le monde remettent plus que jamais en question la portée de sa langue « universelle » et d'une civilisation qui est censée ne l'être pas moins. Longtemps occultée, malgré quelques cris d'alerte ignorés (dont Le Cancer américain, de Robert Aron et Arnaud Dandieu en 1931), la 
montée en puissance des États-Unis d'Amérique - de l'Amérique tout court, en une synecdoque significative - renvoie le pays des droits de l'homme à son statut de puissance très moyenne, au moment même où son Empire se délite, et ravive la hantise du déclin. Bien pire, l'essor de l'anglais et des valeurs de l'American way of life commencent à faire craindre une colonisation culturelle et linguistique. La signature des accords Blum/Byrnes de 1946 provoque un premier grand traumatisme. Ils permettent, comme on le sait, à la cinématographie américaine, dont Truman lui-même avait pourtant dit qu'elle était le fer de lance de la pénétration économique et culturelle américaine, d'envahir les écrans français. À travers elle, malgré les protestations des «intellectuels", se développe dans le grand public un engouement sans précédent pour «l'Amérique» et ses modes dont atteste entre autres la multiplication voyante des emprunts linguistiques à l'anglo-américain.

13 Le livre, la littérature, c'est-à-dire le cœur de la pensée française, sont eux-mêmes atteints et le rôle de Paris en tant que capitale mondiale des Lettres menacé. Dès 1948 sont mises en place de premières mesures, qui instituent auprès du ministère des Affaires étrangères un Comité national du livre français à l'étranger dont les travaux aboutiront à la création du Fonds culturel. De même, la loi de 1949 sur les publications destinées à la jeunesse peut à bien des égards être lue comme une réaction protectionniste face à la pénétration des comics américains et des valeurs qu'ils véhiculent.

14 La Guerre froide avive les tensions. Elle rassemble communistes et compagnons de route dans la dénonciation de l'impérialisme américain, à l'instar de Sartre qui écrit dans Libération, au lendemain de l'exécution des Rosenberg, que "L'Amérique a la rage !». D'autres courants de pensée, toutefois, ne sont pas insensibles à ce discours qui finalement, et jusqu'à aujourd'hui, dépasse les clivages proprement partisans et tisse un lien entre les «nationaux-républicains » de tout bord. En 1953, Alfred Sauvy publie dans Le Monde une tribune intitulée "De l'abandon linguistique à la servitude ", dans lequel il s'alarme du fait que "les Américains manquent rarement une occasion de supplanter non seulement la langue, mais aussi la culture française $»^{17}$.

15 C'est toutefois une dizaine d'années plus tard, au moment même où commence à s'organiser la première francophonie, que les attaques contre «l'ennemi américain », pour reprendre le titre d'un ouvrage de Philippe Roger, atteignent leur acmé, lorsque René Étiemble publie Parlez-vous franglais? On peut certes considérer, comme le fait Bernard Cerquiglini, que « la charmante pochade professorale, cri du cœur d'un savant, est un pamphlet anti-américain haineux et violent, dont les thèmes et la logomachie rappellent les pires officines $»^{18}$. Les excès de ce livre n'en témoignent pas moins d'une position plus ou moins diffuse, non isolée et dont la postérité est assurée par différents écrits, et notamment par L'Aliénation linguistique (1976) et La Guerre culturelle (1979), de Henri Gobard, ou encore par La Colonisation douce, de Dominique Noguez (1991-1993). Si le champ de bataille est principalement linguistique, disent en substance les tenants de cette ligne qui ne répugnent pas au langage guerrier, c'est que la langue gouverne la pensée et que dominer la première revient à diriger la seconde. Se joue donc un conflit de valeurs, un enjeu de civilisation, ne cessent de marteler tant Étiemble que Noguez, dans lequel le «sabir atlantic» joue le rôle du cheval de Troie. Lui ouvrir toutes grandes les portes de la cité revient par conséquent à se laisser envahir et posséder par les valeurs américaines, aux antipodes des idéaux de la Révolution française, le matérialisme et le libéralisme débridés, le commerce et le règne de l'argent, 
l'individualisme, le quantitatif, l'appel à l'impulsion et aux forces obscures, par les diktats de la publicité et du marketing, à l'opposé de la "raison raisonnante », ou encore l'infantilisme, pour ne pas dire l'infantilisation des peuples. Bref, comme l'écrit dans l'esprit Dominique Noguez, l'avoir met l'être à l'étouffoir.

Il s'agit par conséquent pour la pensée française, au-delà de la seule France - et c'est là que la francophonie doit jouer sa partition - de croire qu'elle peut encore proposer au monde un modèle alternatif à l'anti-culture américaine. L'ambition gaulliste, telle que l'expose Michael M. Harrison dans L'Amérique dans les têtes, n'était pas autre :

Comme De Gaulle l'a expliqué une fois à un arabe, la France espérait offrir au monde un modèle permettant de "construire une civilisation industrielle qui ne passe pas par le modèle américain et dans lequel l'homme serait une fin et non un moyen... ». Une certaine fierté de la langue et des coutumes françaises assaillies par la civilisation anglo-saxonne le conduisait à mettre l'accent sur la défense de la langue française et son identité à travers le monde, comme instrument de résistance au pouvoir américain ${ }^{19}$.

\section{Le devenir francophone}

17 Cette rivalité entre deux nations qui, pour reprendre les mots de Marie-France Toinet, « se veulent et se prétendent à la fois universalistes et proposent au monde un modèle éthique $»^{20}$, irrigue-t-elle la francophonie contemporaine ? Un certain nombre de signes permettent de le penser, et notamment l'orientation progressive qu'a prise la Francophonie institutionnelle, de la création de l'ACCT en 1970 aux divers Sommets qui se sont succédé depuis celui de Paris en 1986, en faveur de la diversité culturelle, face aux risques d'uniformisation du monde engendrés par la mondialisation. Le Sommet de Maurice, tout particulièrement, marque l'engagement de la Francophonie dans le combat pour "l'exception culturelle", au moment où se déroulaient, au sein de ce qui n'était encore que le GATT ${ }^{21}$ et dans le cadre du cycle d'Uruguay, des négociations internationales, impulsées par les États-Unis, sur l'élargissement aux services des principes de libéralisation du commerce international qui étaient déjà appliquées aux marchandises. L'opposition des pays francophones, certes, n'a pas empêché l'année suivante, avec la création de l'OMC, l'adoption du $\mathrm{GATS}^{22}$, mais elle a permis pour l'heure d'en entraver l'application concrète et d'écarter de son champ, temporairement, les œuvres cinématographiques et audiovisuelles.

De même, c'est l'action conjuguée des pays francophones, menés par le Canada et la France, qui a permis l'adoption, le 20 octobre 2005, malgré l'opposition des États-Unis, de la Convention «sur la protection et la promotion de la diversité des expressions culturelles », bien que celle-ci, comme le signale Serge Regourd, « reste dépourvue de tout contenu normatif et ne formule aucune disposition de nature à neutraliser les tendances à l'uniformisation inhérentes aux processus de concentration et d'hégémonie des grands groupes économiques $»^{23}$.

19 L'esprit de la francité continuerait-il à privilégier le culturel comme lien social pour manifester une autre manière d'être-au-monde, à l'inverse de l'individualisme et du matérialisme qui sont les deux constituants majeurs de l'anti-américanisme français ?

20 Je me garderai de répondre à cette question. 


\section{NOTES}

1. Cf. Pascal Ory, "Le rôle de l'État: les politiques du livre ", in Histoire de l'édition française (sous la direction de Roger Chartier et Henri-Jean Martin), tome 4, Paris, Fayard/Cercle de la librairie, 1991, p. 65.

2. Cf. Luc Pinhas, «Aux origines du discours francophone: Onésime Reclus et l'expansionnisme colonial français ", Communication \& langages, $n^{\circ}$ 140, juin 2004, p. 69-82.

3. Bernard Cerquiglini, La Naissance du français, Paris, PUF, 1991, p. 120.

4. Le projet de statuts pour l'Académie française remis au cardinal de Richelieu le 22 mars 1634 affirme l'ambition de faire du français « un latin moderne et vivant " (Marc Fumaroli, «La Coupole », Trois Institutions, Paris, Gallimard, coll. Folio, 1994, p. 32-33).

5. Rapporté par Henri Meschonnic, De la langue française, Paris, Hachette, 1997, p. 241.

6. Pierre Bourdieu, "Deux impérialismes de l'universel ", in L'Amérique des Français (sous la direction de Christine Fauré et Tom Bishop), Paris, François Bourin, 1992, p. 149-155.

7. Christophe Traisnel, Francophonie, francophonisme, groupe d'aspiration et formes d'engagement, Paris, université Panthéon-Assas, LGDJ, 1998.

8. Dominique Combes, Poétiques francophones, Paris, Hachette, 1995.

9. Thierry de Beaucé, Nouveau discours sur l'universalité de la langue française, Paris, Gallimard, 1988, p. 77.

10. Cf. Dominique Wolton, «La communication enjeu de deux mondialisations ", in Mondialisation et Francophonie, Montréal, éditions de l'Agence universitaire de la Francophonie, 1998, p. 53-63.

11. Le Plus beau royaume sous le ciel, Paris, 1899 , conclusion.

12. Un grand destin commence, Paris, La Renaissance du livre, 1917, p. 2.

13. Lâchons l'Asie, prenons l'Afrique, p. 151-152.

14. Un grand destin commence, p. 116.

15. Un grand destin commence, titre du chapitre 19.

16. L'assimilation romaine s'est faite, dit Reclus, «par la majesté du nom romain; par la supériorité de la culture; par la diffusion de la langue impériale ; par un réseau serré de routes stratégiques; par un merveilleux souci de l'hygiène des villes; par une admirable utilisation des eaux ; par le respect des institutions locales ; par une tolérance parfaite. Et, en somme, par une patience inaltérable (Un grand destin commence, p. 96 sq.).

17. Alfred Sauvy, « De l'abandon linguistique à la servitude », Le Monde, 6 août 1953.

18. Bernard Cerquiglini, «La révolte des clercs. Estienne, Gourmont, Étiemble contre l"invasion lexicale' ", Mondesfrancophones.com (<http://www.mondesfrancophones.com/ espaces/Frances/articles/revolte-clercs $>$ ).

19. Michael M. Harrison, «La solution gaulliste », in Denis Lacorne, Jacques Rupnik, MarieFrance Toinet (dir.), L'Amérique dans les têtes, un siècle de fascinations et d'aversions, Paris, Hachette, 1986, p. 217.

20. Marie-France Toinet, "L'Antiaméricanisme existe-t-il ? ", in L'Amérique dans les têtes, op. cit., p. 272.

21. General Agreement On Tariffs and Trade.

22. General Agreement On Tariffs and Services.

23. Serge Regourd, "Le livre et l'exception culturelle", Les Cahiers du Syndicat de la librairie française, $\mathrm{n}^{\circ} 4$, avril 2006, p. 12. 


\section{RÉSUMÉS}

La genèse du discours sur la francophonie, des années d'après-guerre aux années 1960, est indéniablement marquée, à tout le moins du point de vue hexagonal, par le sentiment d'un déclin - déchirant à admettre pour la communauté concernée - du rayonnement de la langue française dans le monde et, plus généralement de la culture française. Corrélativement, la montée en puissance, non seulement politique, militaire et économique, mais aussi désormais culturelle, des États-Unis d'Amérique, ne cesse plus à cette époque d'inquiéter les élites françaises, tout autant qu'elle les fascine.

C'est dans ce contexte qu'il convient d'apprécier la résurgence, dans les écrits de Senghor et dans le numéro célèbre de novembre 1962 de la revue Esprit, significativement intitulé «Le français, langue vivante », des termes de " francophone » et de "francophonie ». L'on feint en effet parfois d'oublier que leur apparition, sous la plume d'Onésime Reclus, s'est produite à un autre moment où la France s'interrogeait sur sa grandeur et s'inscrivait dans une réflexion sur la question coloniale, comme l'on ignore trop souvent l'obsession démographique qui présidait à la pensée du géographe. Le recours conjugué à la mythologie "rivarolienne» du génie de la langue française et à la mythologie révolutionnaire du français, langue des droits de l'homme et de l'universel, peut ainsi sembler comme une tentative de redonner son éclat, sinon à la France, du moins à une pensée française qui entend alors manifester clairement sa voix(e) face à celle, grossissante, de « l'Amérique », porteuse d'une autre conception de l'universalité.

Ever since the after-war period and well into the sixties, the whole discourse on "francophonie" has been undeniably flavoured, at least from the French point of view, with the feeling of a decline, a very deplorable thing to admit when seen from Paris, a decline of the radiation of the French language and more generally of the French culture in the world. At the same time, the growing importance of the United States of America, not only politically, economically and in the military field but from now on also culturally, does not stop worrying the French elites, as much as it fascinates them.

The resurgence of such terms as "francophone" and "francophonie" in the writings of Senghor and in the famous November 1962 issue of the review Esprit must be appreciated in this very context. Indeed there is a tendency to forget sometimes that their first use by Onésime Reclus occurred at another time when France was wondering about her "grandeur" and was part of a general reflection on the colonial question, just as one forgets too often the demographic obsession at the origin of the thought of this famous geographer. Thus, the simultaneous recalling of the "rivarolian" myth of the genius of the French language and of the revolutionary myth of French as a language of universality and human rights can look like an attempt at revamping if not France at least a type of French thought, that clearly wants to be heard, in front of the ever-growing "American" thought, which carries with it another type of universality.

\section{INDEX}

Mots-clés : francophonie, déclin, rayonnement, langue française, universalité

Keywords : francophony, decline, influence, French language, universality 
AUTEUR

\section{LUC PINHAS}

Université Paris 13 - Villetaneuse, France

pinhas.luc@wanadoo.fr 